\title{
Sulfur-functionalized Diamond Powder Surface for Attachment of Gold and Biomolecules
}

\author{
Takako Nakamura* and Tsuguyori Ohana \\ National Institute of Advanced Industrial Science and Technology (AIST) \\ Central 5, 1-1-1 Higashi, Tsukuba, Ibaraki 305-8565, Japan \\ * Corresponding author: e-mail: takako-nakamura@aist.go.jp
}

\begin{abstract}
A useful method of modifying the surface of diamond powders with sulfur-containing functionalities was developed by the use of the photolysis of elemental sulfur. The sulfur-modified diamond powder exhibited a surface-attachment behavior to gold nanoparticles through the sulfur-containing linkage. In brief, the exposure of the modified diamond powders to gold colloids resulted in gold nanoparticles being attached to the diamond powders. The introduction of sulfur-containing functional groups and gold nanoparticles on the diamond surfaces was confirmed by means of XPS, DRIFT and mass spectroscopy analyses. Subsequently, the probe DNA attached onto the gold-modified diamond powder showed a DNA hybridization behavior by the treatment with a fluorescence-labeled target DNA oligonucleotide. Key words: diamond powder, chemical modification, sulfur, gold, biomolecules
\end{abstract}

\section{. INTRODUCTION}

Diamond has attracted much attention for use in a ide range of applications because of its unique lectronic, optical and mechanical properties [1]. In articular, diamond powder is widely utilized as an brasive and promote nucleus formation in the abrication of diamond films by chemical vapor eposition (CVD) [2]. The surface modification of iamond powder is expected to lead to the improvement $\mathrm{f}$ its performance, making it possible to introduce novel unctionalities onto the surfaces. Recent studies have zported the introduction of organic functional groups nto the surface of diamond powder using photo- and 1ermochemical methods [3-7].

Organic compounds containing thio- and thiol groups re biologically active substances [8] and show a good ffinity with gold resulting in the formation of a elf-assembled monolayer. Because of the properties of ulfur atoms, the organosulfur chemistry in unctionalized surfaces has been widely investigated in ıe field of self-assembled monolayers, which are mployed effectively as model systems to determine the tructural and chemical properties of organic interfaces nd thin films [9]. Recently, the formation of a iamondoid monolayer on gold film was reported using elf-assembly of thiol-modified diamondoid surfaces 10], however, the preparation of thio-modified iamondoid required multi-step reactions.

Previously we reported that radical reactions assisted y irradiation with UV light led to an effective process or the chemical modification of carbon materials with arious functionalities [11-13]. Commercially available lemental sulfur is easily photolyzed to give orresponding thio-radicals [14]. We report on a onvenient method of modifying the surface of ydrogen-terminated diamond powder with sulfurontaining substituents by the photolysis of elemental ulfur and their attachment behavior to gold and biomolecules.

\section{EXPERIMENTAL}

Elemental sulfur was purchased from Wako Pure Chemical Industries, Ltd. Carbon disulfide and hexane were obtained from Kanto Chemical Co., Inc. Hydrogen-terminated diamond powder was prepared by the hydrogenation of commercially available diamond powder, obtained from Tomei Diamond Co., Ltd. (MD500, particle size $500 \mathrm{~nm}$ ), in accordance with the literature [15]. A gold colloid was also prepared in accordance with the literature [16]. The thiol-modified DNA (S1: HS- $\mathrm{C}_{6} \mathrm{H}_{12}$-T15-GC TTA TCG AGC TTT CG) and oligonucleotides (Cy1: 5'-Cy5 CG AAA GCT CGA TAA GC-3' and Cy2: 5'-Cy5 CG ATT GCT CCT TAA GC-3') labeled with Cy5 fluorescence tags were obtained by Tsukuba Oligo-service, Japan.

Elemental sulfur $(30 \mathrm{mg})$ in carbon disulfide $(4 \mathrm{ml})$ was irradiated using a $20 \mathrm{~W}$ low-pressure mercury lamp (Eikosha EL-A20) through a UV-cut filter $(<200 \mathrm{~nm})$ at room temperature in the presence of hydrogenterminated diamond powder $(30 \mathrm{mg})$ and in an argon atmosphere while stirring. After the reaction mixture was washed ultrasonically with three $2 \mathrm{ml}$ portions of carbon disulfide and hexane then filtered centrifugally to remove the unreacted starting materials, the samples were analyzed by DRIFT, XPS, mass spectroscopy and Raman spectroscopy.

The attachment of gold nanoparticles onto the surface of sulfur-modified diamond powder was carried out by exposing the powder to $0.3 \mathrm{mM}$ gold colloid $(5 \mathrm{ml})$ overnight while stirring. After washing ultrasonically with three $2 \mathrm{ml}$ portions of distilled water and filtering centrifugally to remove the unreacted gold colloid, the samples were analyzed by XPS.

Subsequently, the gold-modified diamond powder was treated with a thiol-modified single-strand probe DNA (S1: $0.13 \mu \mathrm{M}, 1 \mathrm{ml}$ ) by stirring for $7 \mathrm{~h}$ at room 
temperature. After removing the aqueous solution by filtering centrifugally, the diamond powder was washed with water. The following exposure of target (Cy1) and mismatch (Cy2) nucleotides $(50 \mu \mathrm{M}, 1 \mathrm{ml})$ with stirring overnight at room temperature to the probe DNA-treated diamond powder was performed with stirring overnight at room temperature. The samples were analyzed by fluorescence microscopy.

DRIFT spectra were recorded using a JASCO FT/IR-680 Plus spectrometer with a DRIFT attachment (JASCO DR-81). For data evaluation, the KubelkaMunk (KM) transformation was applied to the DRIFT spectra. XPS was carried out by a PHI ESCA model 5800 spectrometer using monochromated aluminum $\mathrm{K} \alpha$ radiation with a pass energy of $23.5 \mathrm{eV}$. Raman spectra were measured with a JASCO NRS-2100 laser Raman spectrometer using the $514.5 \mathrm{~nm}$ line of an argon ion laser with $100 \mathrm{~mW}$ power, and no baseline correction was made. The chemically modified powder was analyzed by a mass spectrometer (JEOL JMSAMSUN200) using the direct insertion method. An Olympus BX51M was applied for the optical and fluorescence microscope analysis.

\section{RESULTS AND DISCUSSION}

3.1 Sulfur-modified diamond powder

The XPS spectra of the diamond powder were measured before and after the treatment with elemental sulfur upon irradiation (Fig. 1a, b). Two new peaks at 165.6 and $228.8 \mathrm{eV}$ of sulfur $2 \mathrm{p}$ and $2 \mathrm{~s}$, respectively, were observed after irradiation together with a carbon peak at $285.0 \mathrm{eV}$. In the carbon $1 \mathrm{~s}$ region, the peak became broad due to the mixture of C-C bonds and C-S bonds with a higher binding energy than that of the hydrogenated diamond powder (Fig. 1c). The deconvoluted of $\mathrm{C} 1 \mathrm{~s}$ spectrum of the sulfur-modified diamond powder has a main peak at $285.0 \mathrm{eV}$ ascribed to the C-C bond together with peaks at 285.8 and 286.2 $\mathrm{eV}$ due to two types of C-S bonds. As shown in Fig. 1d, the deconvoluted S2p spectrum of the modified diamond powder also exhibited two types of $\mathrm{S} 2 \mathrm{p}$ bonds.

Figure 2 shows the DRIFT spectra of hydrogenated diamond powder and diamond powder treated with elemental sulfur after irradiation. The sample after photoreaction has new peaks in the region of 1000-1300 $\mathrm{cm}^{-1}$, attributed to a $\mathrm{C}=\mathrm{S}$ stretching band and $\mathrm{CH}_{2}-\mathrm{S}$ bending vibration, and a decrease in the $\mathrm{C}-\mathrm{H}$ stretching vibration of 2931 and $2834 \mathrm{~cm}^{-1}$ derived from the hydrogenated diamond powder. The results of DRIFT and XPS measurements suggest that the surface photoreaction of hydrogen-terminated diamond powder took place to give sulfur-modified diamond powder with $-\mathrm{SH}$ and $\mathrm{C}=\mathrm{S}$ moieties. The ratio of $\mathrm{C}-\mathrm{SH}$ and $\mathrm{C}=\mathrm{S}$ moieties is $1.0: 1.4$ and $1.0: 1.2$ estimated by XPS $\mathrm{C} 1 \mathrm{~s}$ and $\mathrm{S} 2 \mathrm{p}$ analyses, respectively.

A plausible reaction mechanism as shown in Scheme 1 , is discussed. Polysulfide radicals generated by the ring cleavage of elemental sulfur upon irradiation abstracted hydrogen atoms from the hydrogenated diamond powder to produce polysulfides terminated with both thiol and sulfur radicals, and then the sulfur radicals reacted with the remaining carbon radicals on the diamond surface. The polysulfide bonds $\left(-\mathrm{S}_{\mathrm{n}^{-}}\right)$were easily cleaved to form thiol substituents on the surface
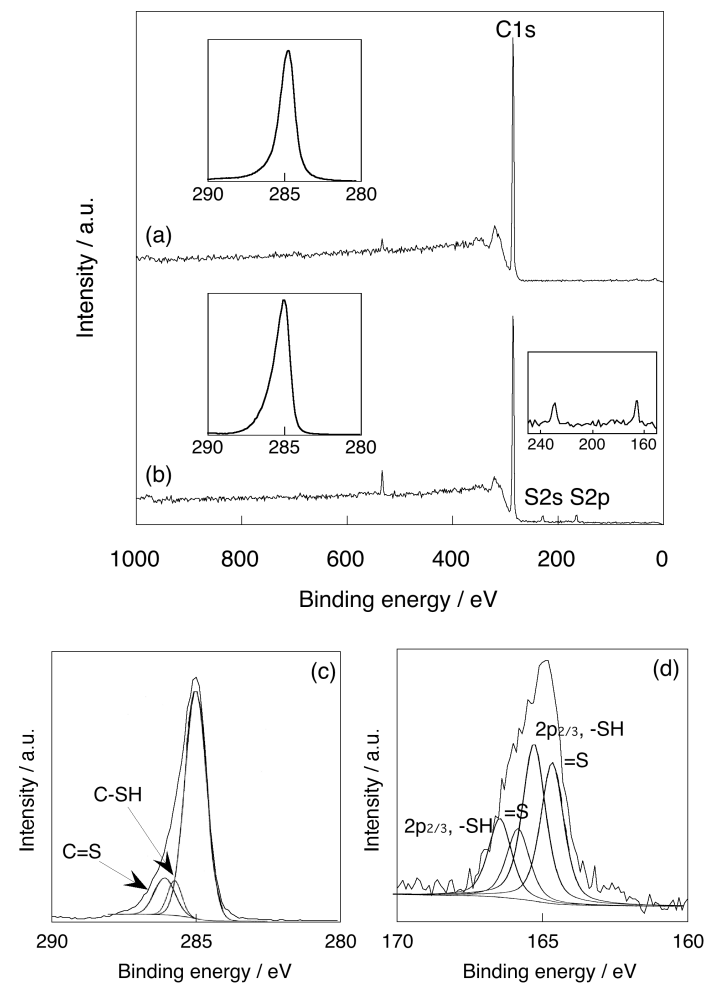

Fig. 1. XPS spectra of (a) hydrogenated diamond powder, (b) diamond powder after irradiation with elemental sulfur, (c) C1s spectrum and (d) S2p spectrum of sulfur-modified diamond powder. The inset shows $\mathrm{C} 1 \mathrm{~s}$ spectra.

via the formation of a thio radical $\mathrm{C}-\mathrm{S}^{\circ}$. In the presence of a methyl group on the diamond surface, hydrogen abstraction and subsequent S-S bond cleavage took place simultaneously to give thiocarbonyl groups via the formation of $-\mathrm{CH}_{2} \mathrm{~S}$ radicals on the surface. This reaction mechanism is supported by a mass spectrum of components generated by heating sulfurized diamond powder to $300{ }^{\circ} \mathrm{C}$, which exhibited two peaks at $\mathrm{m} / \mathrm{z} 32$

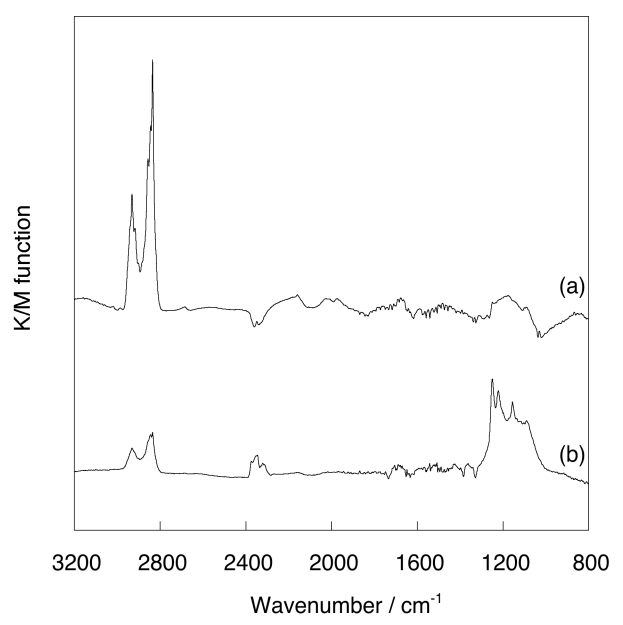

Fig. 2. DRIFT spectra of (a) hydrogenated diamond powder and (b) diamond powder after treatment by sulfur modification. 


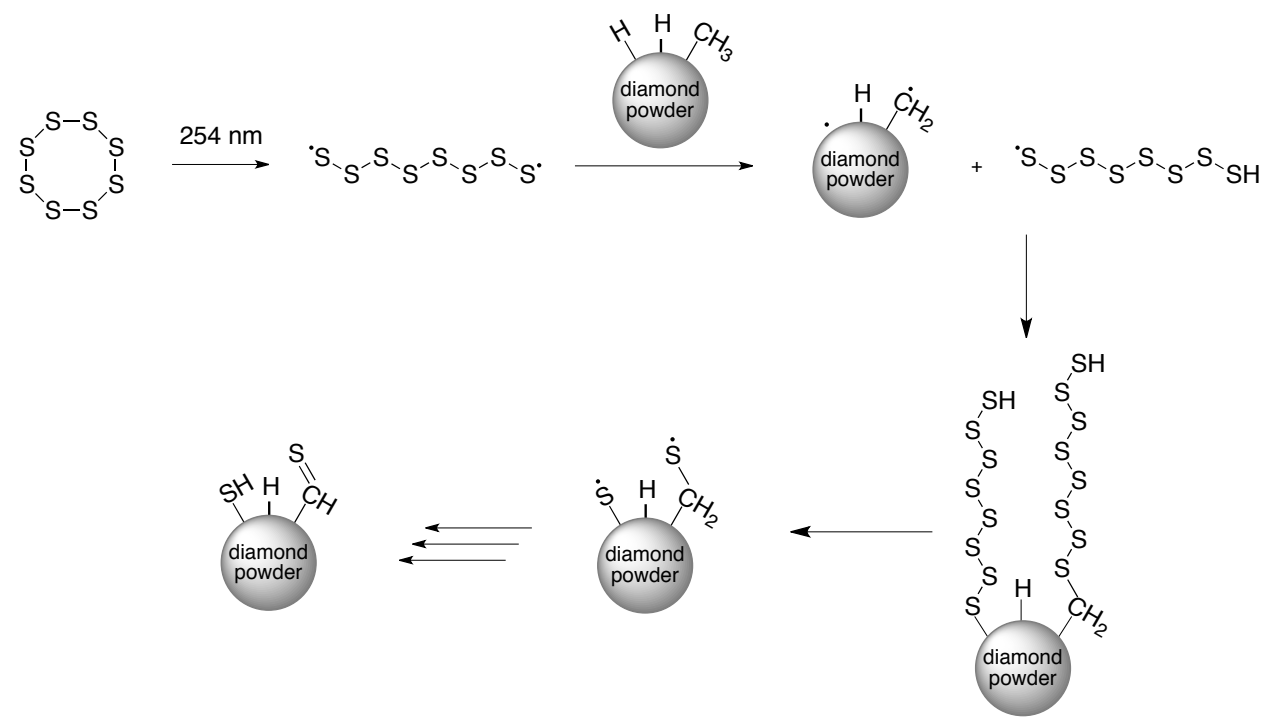

Scheme 1. Chemical modification on the surface of diamond powder with sulfur moieties.

and 44 , ascribed to $-\mathrm{S}$ and $-\mathrm{C}=\mathrm{S}$ groups, respectively (Fig. 3a). Moreover, the mechanism is consistent with the relative bond energies of S-S and C-S bonds of 61-64 and $74 \mathrm{kcal} / \mathrm{mol}$ [17], respectively, meaning that the S-S bonds become weak upon photoirradiation.

The Raman spectra of hydrogenated diamond powder and diamond powder after treatment by irradiation with elemental sulfur were measured as shown in Fig. 3b, c.
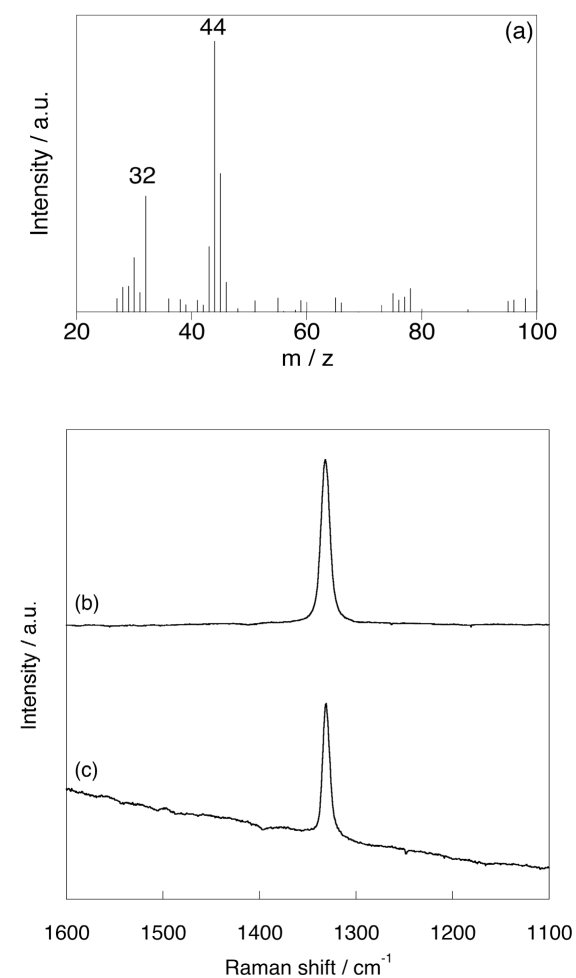

Fig. 3. (a) Mass spectrum of sulfur-modified diamond powders after heating to $300{ }^{\circ} \mathrm{C}$. Raman spectra of (b) hydrogenated diamond powder and (c) diamond powder functionalized with sulfur-containing substituents.
The treated diamond powder exhibited a characteristic diamond peak at $1331 \mathrm{~cm}^{-1}$ similar to that of hydrogenated diamond films at $1333 \mathrm{~cm}^{-1}$. This indicates that the chemical modification of the diamond powder by the photoreaction did not affect its diamond bulk structure. It is known that the fluorescence of samples is the main limitation of Raman scattering experiments due to the generation of a luminescence background. Osticioli et al. reported that the Raman spectra of sulfur compounds show background modulations originating from sulfur atoms [18].

\subsection{Gold-modified diamond powder}

The attachment of gold nanoparticles to sulfurfunctionalized diamond powder was investigated by exposing a gold colloid to modified diamond powder. As shown in Fig. 4, an XPS spectrum of sulfurfunctionalized diamond powder after treatment with the gold colloid exhibits a new peak of Au4f at $84.9 \mathrm{eV}$ together with a C1s peak at $285.0 \mathrm{eV}$ and an S2p peak at $164.7 \mathrm{eV}$. This indicates that gold nanoparticles were

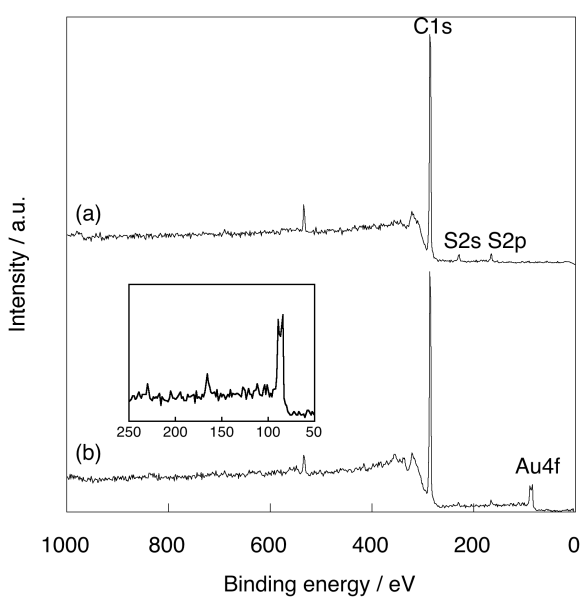

Fig. 4. XPS spectra of sulfur-modified diamond powder after treatment with gold nanoparticles. 
attached to the surface of the diamond powder through thiol and thiocarbonyl linkages by self-assembly.

Since gold nanoparticles attached to the surface of the sulfur-modified diamond powder through sulfurcontaining linkages as described above, a further investigation was made regarding the exposure of the attachment of a thiol-modified probe DNA to the gold-modified diamond powder and the following exposure of target and mismatch DNA with fluorescence tags. As shown in Fig. 5, the fluorescence emission was observed only in the case of the treatment of the target DNA (Cy1), while no emission in the case of the mismatch DNA (Cy2). These results indicate that the gold-modified diamond powder was attached with the probe DNA by self-assembly and will be applicable to a detection agent of biomolecules. The advantage of $\mathrm{Au}$ nanoparticle interlink for DNA attachment is shorter steps from diamond powder to DNA attachment, compared with an ordinary method that needs 4 steps from H-diamond [19].

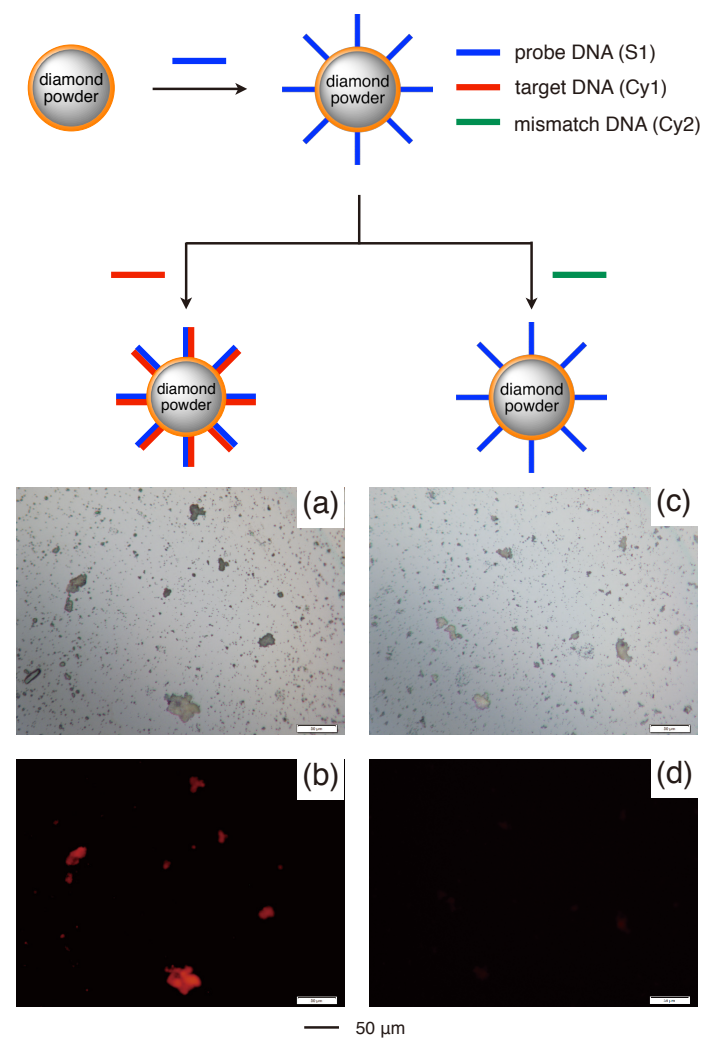

Fig. 5. (a) Optical and (b) fluorescence images of the probe DNA-modified diamond powder after the treatment with a target DNA (Cy1), and (c) optical and (d) fluorescence images with a mismatch DNA (Cy2).

\section{CONCLUSIONS}

The photoreaction of hydrogenated diamond powder in the presence of elemental sulfur is useful for the chemical modification of diamond powder with thiol and thiocarbonyl functional groups using a radical-based process. The modified diamond powder can be attached to a gold nanoparticle surface through sulfur-moiety linkages. Furthermore, the attachment of a thiol-modified probe DNA to the gold-modified diamond powder led to the discrimination of target and mismatch DNA with fluorescence tags. The fluorescence emission was observed only in the case of the treatment of target DNA. The gold-modified diamond powder was found to be applicable to a detection agent of biomolecules by confirming fluorescence analysis.

\section{ACKNOWLEDGMENTS}

This work was supported by a Grant-in-Aid for Scientific Research (C), No. 20560029, from Japan Society for the Promotion of Science (JSPS).

\section{REFERENCES}

[1] J. Wei, V. S. Smentkowski, and J. T. Yates Jr., Crit. Rev. Surf. Chem., 5, 73 (1995).

[2] S. Iijima, Y. Aikawa, and M. Baba, Appl. Phys. Lett., 57, 2646-2648 (1990).

[3] J. B. Miller and D. W. Brown, Langmuir, 12, 5809-5817 (1996).

[4] T. Ando, K. Yamamoto, M. Matsuzawa, Y. Takamatsu, S. Kawasaki, F. Okino, H. Touhara, M. Kamo, and Y. Sato, Diam. Relat. Mater., 5, 1021-1025 (1996).

[5] T. Tsubota, T. Ohno, H. Yoshida, and K. Kusakabe, Diam. Relat. Mater., 15, 668-672 (2006).

[6] T. Nakamura, M. Suzuki, M. Ishihara, T. Ohana, A. Tanaka, and Y. Koga, Langmuir, 20, 5846-5849 (2004).

[7] A. Krueger, J. Mater. Chem., 21, 12571-12578 (2011).

[8] P. Philip, "Organosulfur Chemistry", Academic Press, London (1995).

[9] G. Nagy and A. V. Walker, J. Phys. Chem. B, 110 12543-12554 (2006).

[10] W. L. Yang, J. D. Fabbri, T. M. Willey, J. R. I. Lee, J. E. Dahl, R. M. K. Carlson, P. R. Schreiner, A. A. Fokin, B. A. Tkachenko, N. A. Fokina, W. Meevasana, N. Mannella, K. Tanaka, X. J. Zhou, T. van Buuren, M. A. Kelly, Z. Hussain, N. A. Melosh, and Z. X. Shen, Science, 316, 1460-1462 (2007).

[11] T. Nakamura, Diam. Relat. Mater., 19, 374-381 (2010).

[12] T. Nakamura, T. Ohana, Y. Hagiwara, T. Tsubota, Appl. Surf. Sci., 257, 1368-1370 (2010).

[13] T. Nakamura, T. Ohana, Diam. Relat. Mater., 33 16-19 (2013).

[14] W. Ando, K. Sugimoto, and S. Oae, Bull. Chem. Soc. Jpn., 37, 353-357 (1964).

[15] T. Tsubota, O. Hirabayashi, S. Ida, S. Nagaoka, M. Nagata, Y. Matsumoto, Phys. Chem. Chem. Phys., 4, 806-811 (2002)

[16] K. R. Brown, D. G. Walter, and M. J. Natan, Chem. Mater., 12, 306-313 (2000).

[17] S. Oae, "Organic Chemistry of Sulfur" Ed. by L. Field, Plenum, New York (1977).

[18] I. Osticioli, A. Zoppi, and E. M. Castellucci, Appl. Spectrosc., 61, 839-844 (2007).

[19] W. S. Yang, O. Auciello, J. E. Butler, W. Cai, J. A. Carlisle, J. Gerbi, D. M. Gruen, T. Knickerbocker, T. L. Lasseter, J. N. Russell, L. M. Smith, and R. J. Hamers, Nat. Mater., 1, 253-257 (2002).

(Received February 27, 2013; Accepted May 20, 2013) 